\title{
GEOMETRICALLY FINITE KLEINIAN GROUPS AND PARABOLIC ELEMENTS
}

\author{
by KEN'ICHI OHSHIKA
}

(Received 28th February 1996)

\begin{abstract}
Let $\Gamma$ be a torsion-free geometrically finite Kleinian group. In this paper, we investigate which systems of loxodromic conjugacy classes of $\Gamma$ can be simultaneously made parabolic in a group on the boundary of the quasi-conformal deformation space of $\Gamma$. We shall prove that for this, it is sufficient that the classes of the system are represented by disjoint primitive simple closed curves on the ideal boundary of $\mathbf{H}^{3} / \Gamma$.
\end{abstract}

1991 Mathematics subject classification: 30F40, 57M50.

\section{Introduction}

Let $\Gamma$ be a finitely generated Kleinian group. Let $\Omega_{\Gamma}$ be the region of discontinuity of $\Gamma$ in $S_{\infty}^{2}$. Then, by the Ahlfors finiteness theorem, $\Omega_{\Gamma} / \Gamma$ is a finite union of Riemann surfaces of finite type. Let $Q H_{0}(\Gamma)$ denote the quasi-conformal deformation space of $\Gamma$. Maskit investigated in [15] which systems of loxodromic conjugacy classes of $\Gamma$ can simultaneously be made parabolic in a group on the boundary of $Q H_{0}(\Gamma)$. He proved there that a system of disjoint primitive simple loxodromic boundary conjugacy classes in $\Gamma$ (i.e. distinct primitive loxodromic elements which are represented by disjoint simple closed curves on $\Omega_{\Gamma} / \Gamma$ ) can simultaneously be made parabolic when $\Gamma$ is a geometrically finite function group. On the other hand, in Ohshika [23], we studied the same problem in the case when $\Gamma$ is a geometrically tame Kleinian group which satisfies the condition (*) introduced by Bonahon in [4]. (The condition (*) is equivalent to the indecomposability with respect to free product for Kleinian groups without parabolic elements.) In this paper, we shall study the same problem for general (torsion-free) geometrically finite groups (which may not satisfy the condition (*)). We shall prove that the condition for loxodromic conjugacy classes of $\Gamma$ that they are primitive and represented by disjoint simple closed curves on $\Omega_{\Gamma} / \Gamma$ is sufficient to be made parabolic in a group on the boundary of $Q H_{0}(\Gamma)$ also for general geometrically finite groups. The precise statement is as follows.

Theorem 5.1. Let $\Gamma$ be a geometrically finite Kleinian group. Let $\gamma_{1} \ldots, \gamma_{m}$ be disjoint primitive simple closed curves on $\Omega_{\Gamma} / \Gamma$ representing loxodromic elements in $\Gamma$. Suppose that $\left[\gamma_{j}\right] \neq\left[\gamma_{k}\right]^{ \pm 1}$ if $j \neq k$. Then there exists a geometrically finite Kleinian group $(G, \phi)$ on the boundary of $\overline{Q H_{0}(\Gamma)}$ such that $\left\{\phi\left(\left[\gamma_{1}\right]\right)^{ \pm 1}, \ldots, \phi\left(\left[\gamma_{m}\right]\right)^{ \pm 1}\right\}$ is exactly the set of primitive 
additional parabolic classes, and such that the homotopy equivalence $\phi: \mathbf{H}^{3} / \Gamma \rightarrow \mathbf{H}^{3} / G$ is homotopic to a homeomorphism.

The terms "primitive" and "additional parabolic" will be defined in Section 2.

The key step of the proof of Theorem 5.1 is to prove sequences of quasi-conformal deformations satisfying certain conditions converge in $A H(\Gamma)$. This will be proved by applying Thurston's convergence theorem after proving that the given system of simple closed curves can be extended to one having a property which enables us to apply Thurston's theorem.

We have an alternative proof of Theorem 5.1 depending on the results of [15] and [23] which will not appear in this paper. However, our method in this paper has a merit that it is independent of the results in [15] and [23] except when $\Gamma$ is a Schottky group of rank 2.

Furthermore, Theorem 5.1 can also be proved using Thurston's uniformization theorem and the hyperbolic Dehn surgery theorem instead of Thurston's convergence theorem which we shall make use of in this paper. Compared to this approach, our way to prove Theorem 5.1 has an advantage that we can construct explicitly, by determining the conformal structure at infinity, a sequence converging to the Kleinian group as we want.

Naturally we can ask whether the condition in our theorem is also necessary. To our knowledge, the full solution has not yet been given. A partial solution which deals with the case when $m$, the number of conjugacy classes in the system, is equal to 1 , is given in [25].

Finally, the author would like to express his gratitude to the referee for his/her valuable comments on the original manuscript.

\section{Preliminaries}

A Kleinian group is a discrete subgroup of $P S L_{2} \mathrm{C}$. In this paper we always assume that Kleinian groups are finitely generated and torsion free. A Kleinian group $\Gamma$ acts on $S_{\infty}^{2}$ by conformal automorphisms and on the hyperbolic space $\mathbf{H}^{3}$ by isometries, where $S_{\infty}^{2}$ is the sphere at infinity of the Poincare model of the hyperbolic space. The limit set $\Lambda_{\Gamma}$ in $S_{\infty}^{2}$ is the closure of the set of fixed points (in $S_{\infty}^{2}$ ) of elements (with infinite order) of $\Gamma$. The symbol $\Omega_{\Gamma}$ denotes the region of discontinuity of $\Gamma$ which is the complement of the limit set of $\Gamma$ in $S_{\infty}^{2}$. The Kleinian group $\Gamma$ acts on $\mathbf{H}^{3} \cup \Omega_{\Gamma}$ properly discontinuously. By the Ahlfors finiteness theorem ([2]), the quotient $\Omega_{\Gamma} / \Gamma$ is a (possibly disconnected) Riemann surface of finite type. On the other hand, $\mathbf{H}^{3} / \Gamma$ is a complete hyperbolic 3-manifold. The quotient 3-manifold $\left(\mathrm{H}^{3} \cup \Omega_{\Gamma}\right) / \Gamma$ is called the

Kleinian manifold associated with $\Gamma$.
An element of $P S L_{2} C$ which is conjugate to the matrix $\left(\begin{array}{ll}1 & 1 \\ 0 & 1\end{array}\right)$ is called parabolic. An element of $P S L_{2} \mathrm{C}$ is parabolic if and only if it is not the identity and the absolute value of the trace is equal to 2 . (Note that although the trace has the ambiguity of sign 
for elements in $P S L_{2} \mathrm{C}$, its absolute value is well-defined.) On the other hand, an element which is conjugate to a diagonal matrix $\left(\begin{array}{cc}\lambda & 0 \\ 0 & \lambda^{-1}\end{array}\right)$, where $|\lambda|>1$, is called loxodromic. Any non-trivial element of a torsion-free Kleinian group is either loxodromic or parabolic. By Margulis' lemma, there exists a constant $\epsilon>0$ such that, for any hyperbolic 3-manifold, the set of points where the injectivity radius is less than $\epsilon / 2$ is a disjoint union of two kinds of open sets, one of which is called a Margulis tube, and the other a cusp neighbourhood. A Margulis tube is a tubular neighbourhood of a closed geodesic shorter than $\epsilon$. A cusp neighbourhood, (which is also called simply a cusp), is isometric to the quotient of the subset $\{(x, y, z) \mid z>K\}$ in the upper half space model of the hyperbolic space by either an infinite cyclic group or an abelian group of rank 2 consisting of parabolic isometries fixing the point at infinity $\infty$.

Maximal parabolic subgroups of $\Gamma$ are isomorphic to either $\mathbf{Z}$ or $\mathbf{Z} \times \mathbf{Z}$. Each conjugacy class of a maximal parabolic subgroup corresponds to a cusp of $M=\mathbf{H}^{3} / \Gamma$. For $\epsilon>0$, the $\epsilon$-cusp neighbourhood corresponding to a maximal parabolic group $P$ is the set consisting of points $x \in M$ for which there exist elements of $P$ translating $x$ at distance less than $\epsilon$. Margulis' lemma quoted above claims in particular that if we take a sufficiently small $\epsilon$, then the $\epsilon$-cusp neighbourhoods corresponding to distinct conjugacy classes of maximal parabolic groups are disjoint. We fix such an $\epsilon$ and let $M_{0}$ be the complement of the union of the $\epsilon$-cusp neighbourhoods in $M$.

For a Kleinian group $\Gamma$ and $M=\mathbf{H}^{3} / \Gamma$, the set of homotopic hyperbolic structures preserving cusps in $M$ is denoted by $H(M)$ or $H(\Gamma)$. It is the set consisting of equivalence classes of pairs $(G, \phi)$ where $G$ is a Kleinian group and $\phi$ is a homotopy equivalence from $M$ to $\mathbf{H}^{3} / G$ which maps cusps to cusps. The homotopy equivalences between $M$ and $\mathbf{H}^{3} / G$ correspond bijectively to the isomorphisms from $\Gamma$ to $G$. We use the same symbol as that of a homotopy equivalence to denote the corresponding isomorphism. The condition that a homotopy equivalence $\phi$ maps cusps to cusps is equivalent to one that the isomorphism from $\Gamma$ to $G$ induced by $\phi$ takes parabolic elements of $\Gamma$ to parabolic elements of $G$. Two pairs $\left(G_{1}, \phi_{1}\right)$ and $\left(G_{2}, \phi_{2}\right)$ are regarded as equivalent when the homotopy equivalence $\phi_{2} \circ \phi_{1}^{-1}$ is homotopic to an isometry from $\mathbf{H}^{3} / G_{1}$ to $\mathbf{H}^{3} / G_{2}$, where $\phi_{1}^{-1}$ denotes a homotopy inverse of $\phi_{1}$. Hence the set $H(M)$ can be identified with the set of all faithful discrete representations of $\pi_{1}(M)$ to $P S L_{2} \mathrm{C}$ preserving the parabolicity modulo conjugacy in $P S L_{2} \mathrm{C}$. We can endow $H(M)$ with the topology induced from the topology of the representation space. We call it the algebraic topology, and $A H(M)$ or $A H(\Gamma)$ denotes the space $H(M)$ with the algebraic topology. By Jørgensen's theorem ([12]), the space $A H(\Gamma)$ is closed in the space of representations of $\Gamma$ into $P S L_{2} \mathrm{C}$ modulo conjugacy.

The space of quasi-conformal deformations of $\Gamma$ modulo conjugacy is denoted by $Q H_{0}(\Gamma)$. The set $Q H_{0}(\Gamma)$ can be regarded as a subset of $A H(\Gamma)$. We endow $Q H_{0}(\Gamma)$ with the topology induced from $A H(\Gamma)$. The closure of $Q H_{0}(\Gamma)$ in $A H(\Gamma)$ is denoted by $Q H_{0}(\Gamma)$.

For a hyperbolic 3-manifold $M=\mathrm{H}^{3} / \Gamma$, the convex core of $M$ denoted by $C(M)$ is the minimal convex hyperbolic submanifold of $M$ which is a deformation retract of $M$. A Kleinian group $\Gamma$ is said to be geometrically finite when it is finitely generated and $C\left(\mathbf{H}^{3} / \Gamma\right)$ has finite volume. 
If $\Gamma$ is geometrically finite, $C\left(\mathbf{H}^{3} / \Gamma\right) \cap\left(\mathbf{H}^{3} / \Gamma\right)_{0}$ is compact. In this case, the Kleinian manifold is compactified to a 3 -manifold $\overline{\left(\mathbf{H}^{3} / \Gamma\right)_{0}}$ by attaching annuli and tori, whose interior can be identified with $\left(\mathbf{H}^{3} / \Gamma\right)_{0}$. The intersection $\Omega_{\Gamma} / \Gamma \cap \overline{\left(\mathbf{H}^{3} / \Gamma\right)_{0}}$ is a surface obtained from $\Omega_{\Gamma} / \Gamma$ by removing disjoint neighbourhoods of punctures. (Refer to Chapter 6 of Maskit [17], and Section 6 of Morgan [21].) On the other hand, $\operatorname{Fr}\left(\mathrm{H}^{3} / \Gamma\right)_{0}$ consists of components which are homeomorphic to either a torus or an open annulus. (Here Fr denotes the frontier as a subset in a topological space.) We shall use the symbol $\overline{\mathbf{H}^{3} / \Gamma}$ to denote the Kleinian manifold when $\Gamma$ is geometrically finite. Note that the boundary of $\overline{\mathbf{H}^{3} / \Gamma}$ is equal to the Riemann surface $\Omega_{\Gamma} / \Gamma$.

We use the bracket $[\gamma]$ to denote the conjugacy class of an element $\gamma \in \Gamma$. We also use the symbol $[c]$ for a closed curve $c$ in $\bar{M}$ to denote the conjugacy class represented by $c$ after giving an orientation.

Let $M$ be a 3-manifold with finitely generated fundamental group. A compact 3submanifold of $M$ whose inclusion to $M$ is a homotopy equivalence is called a core of $M$. Peter Scott proved in [26] that for any 3-manifold with finitely generated fundamental group, a core exists. If $M$ is irreducible and the fundamental group of $M$ is indecomposable with respect to free products, then its core is unique up to ambient isotopy. (This follows from the fact that in such a manifold, a core is boundary-irreducible, and homotopic incompressible surfaces are ambient isotopic. Refer also to [20].) When $M$ has a non-empty boundary of finitely many components, all of whose fundamental groups are finitely generated, a core $C$ such that the inclusion of $(C, C \cap \partial M)$ into $(M, \partial M)$ is a homotopy equivalence as pairs, can be chosen as is shown in McCullough [18].

For a compact 3-manifold $N$ with boundary, an embedded torus is said to be essential if it is incompressible and not homotopic to a map into $\partial N$. Similarly, a properly embedded annulus $(A, \partial A) \subset(N, \partial N)$ is said to be essential if it is incompressible and cannot be homotoped to a map into $\partial N$. A compact 3-manifold $N$ is said to be atoroidal if it contains no essential tori. For a Kleinian group $\Gamma$, it is known that $\overline{\left(\mathbf{H}^{3} / \Gamma\right)_{0}}$ is atoroidal. Likewise, any essential torus in $\overline{\mathbf{H}^{3} / \Gamma}$ is homotopic into a cusp neighbourhood corresponding to a maximal parabolic group isomorphic to $\mathbf{Z} \times \mathbf{Z}$. Because of this property, we also say that $\overline{\mathbf{H}^{3} / \Gamma}$ or $\mathbf{H}^{3} / \Gamma$ is "atoroidal" by slightly abusing the term defined as above.

A pair $(M, P)$, where $M$ is a compact irreducible manifold and $P$ is a union incompressible tori and annuli on $\partial M$, is called a pared manifold if (1) every non-cyclic abelian subgroup in $\pi_{1}(M)$ is conjugate to a subgroup of the fundamental group of a torus component of $P$, and (2) there is no essential annulus whose boundary is contained in $P$. This notion of pared manifold is due to Thurston. Refer to [34] and Morgan [21].

\section{Definitions of terms in theorems}

Definition 1. Let $\Gamma$ be a Kleinian group. A closed curve $\gamma$ in $\overline{\mathbf{H}^{3} / \Gamma}$ is said to be primitive when the elements of $\Gamma$ in the conjugacy class represented by $\gamma$ (after giving an orientation on $\gamma$ ) are not non-trivial powers of other elements of $\Gamma$, (in other words, $\gamma$ represents a primitive conjugacy class of $\Gamma$ ). 
Definition 2. Let $\Gamma$ be a Kleinian group. Let $\left(\Gamma^{\prime}, \phi\right)$ be a pair in $A H(\Gamma)$. An element $\gamma$ in $\Gamma^{\prime}$ is said to be an additional parabolic element if it is parabolic and $\phi^{-1}(\gamma)$ is loxodromic. A conjugacy class consisting of (primitive) additional parabolic elements is called a (primitive) additional parabolic class.

Lemma 3.1. Let $c$ be a simple closed curve on $\Omega_{\Gamma} / \Gamma$ for a Kleinian group $\Gamma$, which represents a loxodromic conjugacy class of $\Gamma$. Then, $c$ is primitive if and only if the following condition is satisfied.

(The condition): There is no essential annulus properly embedded in $\left(\overline{\mathbf{H}^{3} / \Gamma}, \Omega_{\Gamma} / \Gamma\right)$ both of whose boundary components are homotopic on $\Omega_{\Gamma} / \Gamma$ to the simple closed curve $c$.

Proof. Suppose that $c$ does not satisfy the condition in the lemma. Then there exists a properly embedded essential annulus $A$ such that each component of $\partial A$ is parallel to $c$. Let $B$ be an annulus on $\Omega_{\Gamma} / \Gamma$ bounded by $\partial A$ which contains $c$. Then the torus $A \cup B$ bounds a solid torus $V$ because $\bar{M}$ is atoroidal and $A$ contains the curve $c$ representing a loxodromic class of $\Gamma$, where $M=\mathrm{H}^{3} / \Gamma$. Since $A$ is not homotopic to $B$ in $V$, the core curve of $B$ represents the $p$-th power with $p \geq 2$ of a generator of $\pi_{1}(V) \cong \mathbf{Z}$. As $c$ is homotopic to the core curve of $B$, it represents a non-primitive conjugacy class of $\Gamma$. Hence the "only if" part is proved.

Conversely, suppose that $c$ is not primitive. Then there exists a simple closed curve $\gamma$ in $M$ and an integer $p \geq 2$ such that the $p$-time iteration of $\gamma$ is freely homotopic to $c$. Let $A$ be a singular annulus realizing a homotopy between $c$ and $\gamma^{p}$ where the pth power denotes the $p$-time iteration. Let $V$ be a thin tubular neighbourhood of $\gamma$. We can homotope $A$ so that it intersects $\partial V$ near the component of $\partial A$ corresponding to $\gamma^{p}$ at a simple closed curve $\delta$ on $\partial V$ wrapping $p$ times around $\gamma$.

By thickening $A$, we can construct a map $F: S^{1} \times[0,1] \times[0,1] \rightarrow \bar{M}$, where $S^{1}$ is a circle, which is transverse to $\partial V$, such that $F\left(S^{1} \times[0,1] \times\{0.5\}\right)=A, F\left(S^{1} \times\{0\} \times[0,1]\right)$ is a thin annulus neighbourhood of $c$ in $\Omega_{\Gamma} / \Gamma, F\left(S^{1} \times\{1\} \times[0,1]\right)=\gamma^{p}$, and such that for a simple closed curve $d$ on $S^{1} \times[0,1]$ which is mapped to $\delta$ by $F \mid\left(S^{1} \times[0,1] \times\{0.5\}\right)$, the image $F(d \times[0,1])$ is an annulus neighbourhood $\Delta$ of $\delta$ in $\partial V$. Let $A^{\prime}$ be the annulus on $S^{1} \times[0,1]$ bounded by $S^{1} \times\{0\}$ and $d$. Let $\Delta^{\prime}$ be the annulus $\partial V-\operatorname{Int} \Delta$.

Consider the singular annulus $F\left(A^{\prime} \times\{0,1\}\right) \cup \Delta^{\prime}$, which we shall denote by $A^{\prime \prime}$. Suppose that $A^{\prime \prime}$ is homotopic into the boundary of $\bar{M}$ preserving the boundary, which means that $A^{\prime \prime}$ is homotopic to the annulus neighbourhood $F\left(S^{1} \times\{0\} \times[0,1]\right)$ fixing the boundary. Since $F\left(A^{\prime} \times\{0,1\}\right) \cup \Delta$ is homotopic to $F\left(S^{\prime} \times\{0\} \times[0,1]\right)$ by definition, the two annuli $\Delta$ and $\Delta^{\prime}$ must be homotopic relatively to the boundary then. This cannot happen since we assumed $p \geq 2$. Thus we have proved that $A^{\prime \prime}$ is essential (or one says non-degenerate in some references).

By the annulus theorem proved in Jaco-Shalen [11], there exists an essential embedded annulus $B$ such that $\partial B=\partial A^{\prime \prime}$. This means that $c$ does not satisfy the condition, and the proof of the "if" part is completed.

We can further prove the following corollary by refining the proof of the lemma above a little. 
Corollary 3.2. Let $c$ be a simple closed curve on $\Omega_{\Gamma} / \Gamma$ representing a loxodromic conjugacy class for a Kleinian group $\Gamma$ as before. Suppose that $c$ is not primitive. Then there exists an embedded essential annulus $B$ in $\overline{\mathrm{H}^{3} / \Gamma}$ both of whose boundary components are parallel to $c$ on $\Omega_{\Gamma} / \Gamma$. Furthermore, the annulus $B$ cuts off a solid torus from $\overline{\mathbf{H}^{3} / \Gamma}$.

Proof. We have proved that an embedded annulus $B$ as in the statement exists in the proof of Lemma 3.1. We shall prove that $B$ cuts off a solid torus from $\overline{\mathbf{H}^{3} / \Gamma}$. Recall that $\partial B$ bounds an annulus $B^{\prime}$ on $\Omega_{\Gamma} / \Gamma$, which is a neighbourhood of $c$. Since $\overline{\mathbf{H}^{3} / \Gamma}$ is atoroidal and $c$ represents a loxodromic class, the torus $B \cup B^{\prime}$ must bound a solid torus in $\overline{\mathbf{H}^{3} / \Gamma}$. This completes the proof.

\section{Extending a system of simple closed curves}

In this section, we shall prove the following proposition which will be used to prove Theorem 5.1.

Proposition 4.1. Let $\Gamma$ be a geometrically finite Kleinian group with $M=\mathbf{H}^{3} / \Gamma$. Let $\gamma_{1}, \ldots, \gamma_{m}$ be a system of disjoint primitive simple closed curves each of which lies on either $\Omega_{\Gamma} / \Gamma \cap \bar{M}_{0}$ or an annulus component of $\operatorname{Fr} M_{0}=\partial \bar{M}_{0}-\Omega_{\Gamma} / \Gamma$, and is essential in $\bar{M}_{0}$, such that after giving an orientation on each $\gamma_{i}$, we have $\left[\gamma_{i}\right] \neq\left[\gamma_{j}\right]^{ \pm 1}$ for $i \neq j$ in $\pi_{1}\left(\bar{M}_{0}\right)=\Gamma$. Suppose that a core curve of each annulus component of $\operatorname{Fr} M_{0}$ is contained in the family $\gamma_{1} \ldots \gamma_{m}$. Then there exist disjoint simple closed curves $\bar{\gamma}_{1}, \ldots, \bar{\gamma}_{n}$ each of which lies either on $\Omega_{\Gamma} / \Gamma \cap \bar{M}_{0}$ or an annulus component of $\operatorname{Fr} M_{0}$, and is essential in $\bar{M}_{0}$ such that the following four conditions are satisfied.

(1) For $j=1, \ldots, m$ the equality $\bar{\gamma}_{j}=\gamma_{j}$ holds.

(2) Every component of $\partial \bar{M}_{0}-\cup_{j} \bar{\gamma}_{j}$ that is not a torus corresponding to $\mathbf{Z} \times \mathbf{Z}$-cusp is homeomorphic to a thrice-punctured sphere.

(3) For each different number $j, k$, if $\left[\left(\bar{\gamma}_{j}\right)\right]^{p}=\left[\left(\bar{\gamma}_{k}\right)\right]^{q}$ for some integers $p, q$, it follows that $p=q=0$, where [] denotes the conjugacy class as before and the pth power of a conjugacy class means the conjugacy class consisting of the pth powers of elements in the original conjugacy class, (which is evidently uniquely determined).

(4) For each $j>m$, the closed curve $\bar{\gamma}_{j}$ represents a loxodromic class of $\Gamma$.

Proof. By enlarging $\gamma_{1}, \ldots, \gamma_{m}$, we can construct a family of simple closed curves $\gamma_{1}^{0}, \ldots, \gamma_{n}^{0}$ each of which lies on either $\Omega_{\Gamma} / \Gamma \cap \bar{M}_{0}$ or an annulus component of $\operatorname{Fr} M_{0}$ satisfying the two conditions (1), (2) in the proposition i.e., such that $\gamma_{j}^{0}=\gamma_{j}$ for $j=1, \ldots, m$ and each non-torus component of $\partial \bar{M}_{0}-U_{j=1}^{n} \gamma_{j}^{0}$ is homeomorphic to a thrice-punctured sphere. Let $\gamma_{1}^{1}, \ldots, \gamma_{n}^{1}$ be a family of simple closed curves satisfying the conditions (1), (2) such that the number of simple closed curves in the family which are null-homotopic in $\bar{M}_{0}$ is minimal among such families. We shall construct $\bar{\gamma}_{1}, \ldots, \bar{\gamma}_{n}$ starting from this family $\gamma_{1}^{1}, \ldots, \gamma_{n}^{1}$ and modifying it step by step. 
First, let us prove that there is no simple closed curve in $\left\{\gamma_{1}^{1}, \ldots, \gamma_{n}^{1}\right\}$ which is null-homotopic in $\bar{M}_{0}$ by contradiction. Suppose that there exists some $\gamma_{k}^{1}$ which is null-homotopic in $\bar{M}_{0}$. Then $\gamma_{k}^{1}$ bounds a compressing disc $\Delta$ in $\bar{M}_{0}$ by the loop theorem (which is due to Stallings [28].) Let $P$ be the component of $\partial \bar{M}_{0}-U_{j \neq k} \gamma_{j}^{\prime}$ containing $\gamma_{k}^{1}$. Then $P$ is homeomorphic to either a once-punctured torus or a fourtime-punctured sphere.

Suppose first that $P$ is homeomorphic to a once-punctured torus. Let $\delta$ be an essential simple closed curve in $P$ such that $i\left(\delta, \gamma_{k}^{l}\right)=1$. (We use the symbol $i$ for denoting the geometric intersection number from now on, i.e., for closed curves $\alpha, \beta$ on a surface, the symbol $i(\alpha, \beta)$ denotes the minimal number of intersections between $\alpha$ and a closed curve freely homotopic to $\beta$, which we can express as $\min _{b \approx \beta} \#\{\alpha \cap b\}$.) Then $\delta$ represents a homologically non-trivial element of $\pi_{1}(M)$ by the Poincaré duality theorem. By replacing $\gamma_{k}^{1}$ by $\delta$, we can reduce the number of simple closed curves in the family which are null-homotopic in $\bar{M}_{0}$. (Note that $\gamma_{k}^{1}$ is not one of $\gamma_{1}, \ldots, \gamma_{m}$ as it is null-homotopic in $\bar{M}_{0}$.) This contradicts our choice of $\gamma_{1}^{1}, \ldots, \gamma_{n}^{1}$.

Next suppose that $\boldsymbol{P}$ is homeomorphic to a four-times-punctured sphere. Let $\delta$ be an essential simple closed curve in $P$ such that $i\left(\delta, \gamma_{k}^{1}\right)=2$. If $\delta$ is essential in $\bar{M}_{0}$, by replacing $\gamma_{k}^{1}$ with $\delta$, we get a contradiction as before. Suppose that $\delta$ is also null-homotopic, hence bounds a compression disc $D$. By a standard cut-and-paste technique (refer for instance to Jaco [10]), we can assume that $D \cap \Delta$ consists of only one arc dividing $D$ into $D_{1}$ and $D_{2}$, and $\Delta$ into $\Delta_{1}$ and $\Delta_{2}$. Then by pasting $D_{\alpha}$ and $\Delta_{\beta}$ for $\alpha, \beta=1,2$, we can see that each component of FrP bounds a compression disc. To sum up, if $\gamma_{k}^{1}$ is null-homotopic in $\bar{M}_{0}$, it must be contained in a component $P$ of $\partial \bar{M}_{0}-U_{j \neq k} \gamma_{j}^{\prime}$ which is homeomorphic to a four-timespunctured sphere, and all the simple closed curves in $\left\{\gamma_{1}^{1}, \ldots, \gamma_{n}^{1}\right\}$ which are the frontier components of $P$ must bound compression discs.

Let $S$ be a component of $\partial \bar{M}_{0}$ on which $\gamma_{k}^{\prime}$ as above lies. By the argument in the last paragraph, each simple closed curve in $\left\{\gamma_{1}^{1}, \ldots, \gamma_{n}^{1}\right\}$ which lies on $S$ must be contained in a sphere with four holes all of whose boundary components are in $\left\{\gamma_{1}^{1}, \ldots, \gamma_{n}^{1}\right\}$ and null-homotopic in $\bar{M}_{0}$. This is possible only when all the simple closed curves on $S$ that are contained in $\left\{\gamma_{1}^{1}, \ldots, \gamma_{n}^{1}\right\}$ bound compression discs. In particular, since $\gamma_{1}^{1}, \ldots, \gamma_{m}^{1}$ do not bound compressing discs, none of them lie on $S$. On the other hand, it is clear that we can choose simple closed curves on $S$ at least one of which is essential in $\bar{M}_{0}$ and whose complement is a disjoint union of thrice-punctured spheres. By replacing the simple closed curves in the family $\gamma_{1}^{1}, \ldots, \gamma_{n}^{1}$ on $S$ with these simple closed curves, we can reduce the number of null-homotopic simple closed curves in the family, and get a contradiction. Thus we have proved that no simple closed curves in $\gamma_{1}^{1}, \ldots, \gamma_{m}^{1}$ are null-homotopic in $\bar{M}_{0}$.

Next we shall show that we can arrange $\gamma_{1}^{1}, \ldots, \gamma_{n}^{1}$ so that if $\left[\left(\gamma_{k}^{1}\right)\right]^{p}=\left[\left(\gamma_{i}^{1}\right)\right]^{q}$ for some $k \neq l$, then we should have $p=q=0$, by replacing $\gamma_{j}^{1}$ for $j>m$. Before that, let us prove that we can arrange $\gamma_{1}^{1}, \ldots, \gamma_{n}^{1}$ so that they should represent different conjugacy classes of $\pi_{1}(M)$ no matter which orientation we choose for them. In other words, we can arrange them so that no two of $\gamma_{1}^{1}, \ldots, \gamma_{n}^{1}$ are freely homotopic in $\bar{M}_{0}$. Suppose that some $\gamma_{k}^{1}$ and $\gamma_{1}^{1}$ with $k \neq l$ represent the same conjugacy class of $\pi_{1}(M)$ after choosing appropriate orientations. 
As $\gamma_{1}^{1}, \ldots, \gamma_{m}^{1}$ represent different conjugacy classes, we can assume that $k>m$. For $\gamma_{k}^{1}$, define $P$ and $\delta$ as before. (Recall that there are two possibilities; $P$ is homeomorphic to either a once-punctured torus or a four-times-punctured sphere.) If none of the $\gamma_{j}^{1}$ 's $(j=1, \ldots, n ; j \neq k)$ represent the same element as the one which $\delta$ represents (after choosing an appropriate orientation), and $\delta$ is essential in $\bar{M}_{0}$, we can replace $\gamma_{k}^{1}$ by $\delta$ and reduce the number of the pairs which represent a common conjugacy class of $\Gamma$.

Suppose that there exists $\gamma_{l^{\prime}}^{1}\left(l^{\prime} \neq k\right)$ which represents the same conjugacy class of $\Gamma$ as the one which $\delta$ represents. Then by the generalized Dehn's lemma in ShapiroWhitehead [27], there exist properly embedded essential annuli $A$ and $A^{\prime}$ in $\bar{M}_{0}$ such that $\partial A=\gamma_{k}^{1} \cup \gamma_{l}^{1}$, and $\partial A^{\prime}=\delta \cup \gamma_{p}^{1}$. We can assume that $A$ and $A^{\prime}$ intersect transversely. If $l=l^{\prime}$, we isotope $\partial A^{\prime}$ slightly so that the boundary component of $A^{\prime}$ corresponding to $\gamma_{i}^{1}$ and that of $A$ corresponding to $\gamma_{l}^{1}$ are disjoint. Then since the boundary components of $A$ and $A^{\prime}$ corresponding to $\gamma_{t}^{1}$ and $\gamma_{1}^{1}$, respectively are disjoint anyway, and $\partial A$ and $\partial A^{\prime}$ cannot be disjoint, there must be an arc $\alpha$ in $A \cap A^{\prime}$ such that $\partial \alpha \subset \gamma_{k}^{1} \cap \delta$. This is impossible if $P$ is homeomorphic to a once-punctured torus. Hence we can assume that $\boldsymbol{P}$ is homeomorphic to a four-times-punctured sphere. Furthermore as $i(\delta, \gamma)=2$ in this case, we have $\partial \alpha=\delta \cap \gamma_{k}^{1}$. Since both the points of $\partial \alpha$ are on the same component of $\partial A$ and $\partial A^{\prime}$, the arc $\alpha$ bounds outermost semi-discs $\Delta$ and $\Delta^{\prime}$ respectively in $A$ and $A^{\prime}$ relatively to $\partial A$ and $\partial A^{\prime}$ respectively. By a well-known cut-andpaste technique, we can assume that Int $\Delta \cap \operatorname{Int} \Delta^{\prime}=\emptyset$. Then $\Delta \cup \Delta^{\prime}$ is isotopic to a disc bounded by some component of FrP. This contradicts the fact that none of the $\gamma_{j}$ 's bound a compression disc.

Next suppose that $\delta$ is null-homotopic in $\bar{M}_{0}$. Then $\delta$ bounds a compression disc $D$ in $\bar{M}_{0}$. We can assume that $D$ and $A$ meet each other transversely. If $P$ is homeomorphic to a once-punctured torus, $\partial D \cap \partial A$ consists of one point. However, this cannot happen because then, the two components of $\partial A$, which are obviously homologous in $\bar{M}_{0}$, would have distinct algebraic intersection numbers with $\delta$. If $P$ is homeomorphic to a four-times-punctured sphere, we have $i(\partial D, \partial A)=2$. Then there must be an arc $\alpha$ in $D \cap A$ such that $\partial \alpha=\delta \cap \gamma_{k}^{\prime}$. The arc $\alpha$ separates a semi-disc $\Delta$ from $A$, and cuts $D$ into two semi-discs $D_{1}$ and $D_{2}$. By removing circle components of $D \cap A$ by a well-known cut and paste technique, we can assume that the interior of $D_{1}$ does not intersect $A$. Then $\Delta \cup D_{1}$ is isotopic to an embedded compressing disc for one of the components of $\operatorname{Fr} P$. This contradicts the fact that each of $\gamma_{1}^{1}, \ldots, \gamma_{n}^{1}$ is essential in $\bar{M}_{0}$. Thus we can reduce the number of pairs in $\gamma_{1}^{1}, \ldots, \gamma_{n}^{1}$ representing a common conjugacy class, and by induction, we get $\gamma_{1}^{1}, \ldots, \gamma_{n}^{1}$ satisfying the conditions (1), (2), which does not contain such a pair.

Next suppose that some $\left[\left(\gamma_{k}^{1}\right)\right]^{p}$ and $\left[\left(\gamma_{l}^{1}\right)\right]^{q}$ are equal for $k \neq l$ for $\gamma_{1}^{1}, \ldots, \gamma_{n}^{1}$ which are modified as above. Suppose that neither $p$ nor $q$ is equal to 0 . (We have already proved that if $p=0$ or $q=0$, then so is the other.) By assumption, such a situation cannot occur if $k, l \leq m$. Therefore we can assume that $k>m$. Let $P$ and $\delta$ be as before for $\gamma_{k}^{1}$. If none of the $\left[\left(\gamma_{j}^{\prime}\right)\right]^{q^{\prime}}$ 's for $q^{\prime} \neq 0$ and $j=1, \ldots n(j \neq k)$ coincide with $[\delta]^{p^{\prime}}$ for $p^{\prime} \neq 0$, and $\delta$ is essential in $\bar{M}_{0}$, we replace $\gamma_{k}^{1}$ by $\delta$ and can reduce a pair which violates the condition (3) in our proposition. 
Suppose that there exists $\gamma_{i}^{1}\left(l^{\prime} \neq k\right)$ such that $\left[\left(\gamma_{l}^{l}\right)\right]^{q^{\prime}}=[\delta]^{\prime}$ for non-zero $p^{\prime}$ and $q^{\prime}$. (This assumption implies in particular that $\delta$ is essential in $\bar{M}_{0}$.) Then there exist singular annuli $A$ and $A^{\prime}$ such that $\partial A$ is a union of the $p$-time iteration of $\gamma_{k}^{1}$ and the $q$-time iteration of $\gamma_{l}^{\prime}$ and $\partial A^{\prime}$ is a union of the $p^{\prime}$-time iteration of $\delta$ and the $q^{\prime}$-time iteration of $\gamma_{l}^{\prime}$. By homotoping $A$ and $A^{\prime}$, we can assume that they are self-transverse immersions and meet each other transversely keeping their boundaries in small neighbourhoods of the original boundaries. Moreover, we can assume that there are no inessential intersections between $\partial A$ and $\partial A^{\prime}$ on $\partial \bar{M}_{0}$. (Here we slightly abuse the notation and use the symbol $A$ or $A^{\prime}$ to denote both the annulus as a map and its image.) Let $a$ be a smooth arc in $A \cap A^{\prime}$. Note by the same argument as in the last paragraph, we can assume that $\partial a$ lies on the boundary component of $\partial A$ corresponding to $\left(\gamma_{k}^{1}\right)^{p}$, and that of $\partial A^{\prime}$ corresponding to $\delta^{p^{\prime}}$. Then there exist arcs $b$ on $\partial A$ and $b^{\prime}$ on $\partial A^{\prime}$ such that $a \cup b$ bounds an immersed semi-disc on $A$, and $a \cup b^{\prime}$ bounds one on $A^{\prime}$. The closed arc $b \cup b^{\prime}$ is essential in $P$ because of the minimality of the intersection between $\partial A$ and $\partial A^{\prime}$, and bounds a singular disc in $\bar{M}_{0}$. Therefore, by the loop theorem, there exists an essential simple closed curve $c$ in $P$ which bounds a compression disc in $\bar{M}_{0}$. It follows that when $P$ is homeomorphic to a four-times-punctured sphere, either some component of $\operatorname{Fr} P$ bounds a disc or some two components of $\operatorname{Fr} P$ are homotopic in $\bar{M}_{0}$, which contradicts the fact that none of $\gamma_{1}^{1}, \ldots, \gamma_{n}^{1}$ bound dises and no two simple closed curves of $\gamma_{1}^{1}, \ldots, \gamma_{n}^{1}$ are homotopic. When $P$ is homeomorphic to a once-punctured torus, there must be a disc $D$ whose boundary is a simple closed curve in $P$ which is essential relative to $\operatorname{Fr} P$. Then $I\left(D,\left[\left(\gamma_{k}^{1}\right)\right]^{p}\right) \neq 0$, where $I$ denotes the algebraic intersection number. (Note that the algebraic intersection number depends only on the homology class, hence we need not care about the choice of a representative in a conjugacy class.) On the other hand, as $\gamma_{i}^{\prime}$ is disjoint from $P$, we have $I\left(D,\left[\left(\gamma_{l}^{\prime}\right)\right]^{9}\right)=0$, and it is a contradiction.

If $\delta$ is null-homotopic in $\bar{M}_{0}$, a similar argument can be applied for a disc $D$ bounded by $\delta$ and the annulus $A$ instead of $A$ and $A^{\prime}$ in the above, and by the same argument, we get a contradiction. Therefore we can reduce the number of pairs in $\gamma_{1}^{1}, \ldots, \gamma_{n}^{1}$ such that $\left[\left(\gamma_{k}^{1}\right)\right\}^{p}=\left[\left(\gamma_{j}^{1}\right)\right]^{q}$ for non-zero $p, q$ preserving the conditions (1), (2). Thus we obtain a family of simple closed curves $\gamma_{1}^{1}, \ldots, \gamma_{n}^{1}$ essential in $\bar{M}_{0}$ which satisfies the conditions (1), (2), (3).

Finally, let us prove that we can arrange $\gamma_{1}^{1}, \ldots, \gamma_{n}^{1}$ so that each $\gamma_{j}^{1}$ which is not in the original family $\gamma_{1}, \ldots, \gamma_{m}$ represents a loxodromic class. Since a core curve of each annulus component of $\operatorname{Fr} M_{0}$ is contained in the original family, by the condition (3), if some $\gamma_{j}^{1}$ represents a parabolic class, it must be freely homotopic to a closed curve $k$ on a torus component of $\operatorname{Fr} M_{0}$.

Consider $P \subset \partial \bar{M}_{0}$ which contains $\gamma_{f}^{1}$ in its interior and a simple closed curve $\delta \subset P$ as before. Then by the same argument as before, we can prove that $\delta$ must be essential in $\bar{M}_{0}$, and that no non-trivial power of $\delta$ is freely homotopic to a power of $\gamma_{i}^{1}$ with $i \neq j$. Therefore if $\delta$ represents a loxodromic element we can simply replace $\gamma_{j}^{l}$ with $\delta$ and reduce the number of curves in $\gamma_{1}^{1}, \ldots, \gamma_{n}^{1}$ representing a parabolic class preserving the conditions (1)-(3). Furthermore by considering annuli in a way similar to the preceding argument, we can prove that even if $\delta$ also represent a parabolic class, it is impossible that $\gamma_{j}^{l}$ and $\delta$ are homotopic to closed curves in distinct torus components of $\operatorname{Fr} M_{0}$. 
Thus the only case that we have to consider is when there are two closed curves $k, l$ on a torus component of $\operatorname{Fr} M_{0}$ such that $\gamma_{j}^{1}$ is freely homotopic to $k$ and $\delta$ to $l$. We can consider annuli realizing free homotopy from $\gamma_{j}^{l}$ to $k$, and from $\delta$ to $l$, and by the argument as before using the property that no components of $\operatorname{Fr} P$ are null-homotopic in $\bar{M}_{0}$, we can see that $k$ and $l$ must intersect essentially, and that the annuli must meet each other at an arc $a$ which connects the two boundary components of them. Take a base point $x$ on $P \cap a$ and consider the elements $\kappa, \lambda$ of $\pi_{1}\left(\bar{M}_{0}, x\right)$ represented by $a k a^{-1}$ and $a l a^{-1}$ respectively. Then $\kappa$ is also represented by the loop $\gamma_{j}^{1}$ and $\lambda$ by $\delta$. The elements $\kappa$ and $\lambda$ commute in $\pi_{1}\left(\bar{M}_{0}\right)$ because $k$ and $l$ lies on the same torus. This implies that $\pi_{1}(P)$ does not inject into $\pi_{1}\left(\bar{M}_{0}\right)$.

It follows by the loop theorem that there exists either a component of $\operatorname{Fr} P$ which is null-homotopic in $\bar{M}_{0}$, or a simple closed curve $\delta^{\prime}$ on $P$ which is null-homotopic in $\bar{M}_{0}$ and intersects $\gamma_{j}^{1}$ essentially. The former cannot exist because none of $\gamma_{1}^{1}, \ldots, \gamma_{n}^{1}$ are null-homotopic in $\bar{M}_{0}$. In the latter case, we can apply the argument as before considering the intersection between a compression disc bounded by $\delta^{\prime}$ and a singular annulus bounded by $\gamma_{j}^{1}$ and $k$, and get a contradiction.

Thus we have proved that the simple closed curve $\delta$ must represent a loxodromic class. By replacing all the curves in $\left\{\gamma_{1}^{1}, \ldots, \gamma_{n}^{l}\right\} \backslash\left\{\gamma_{1}, \ldots, \gamma_{m}\right\}$ that represent a parabolic class with curves representing loxodromic class by this fashion, we get a family which satisfies all the conditions (1)-(4). This completes the proof.

\section{Construction of the limit group}

The following is our main theorem whose proof we shall complete in this section.

Theorem 5.1. Let $\Gamma$ be a geometrically finite Kleinian group. Let $\gamma_{1} \ldots, \gamma_{m}$ be disjoint primitive simple closed curves on $\Omega_{\Gamma} / \Gamma$ representing loxodromic elements in $\Gamma$. Suppose that $\left[\gamma_{j}\right] \neq\left[\gamma_{k}\right]^{ \pm 1}$ if $j \neq k$. Then there exists a geometrically finite Kleinian group $(G, \phi)$ on the boundary of $\overline{Q H_{0}(\Gamma)}$ such that $\left\{\phi\left(\left[\gamma_{1}\right]\right)^{ \pm 1}, \ldots, \phi\left(\left[\gamma_{m}\right]\right)^{ \pm 1}\right\}$ is exactly the set of primitive additional parabolic classes, and such that the homotopy equivalence $\phi: \mathbf{H}^{3} / \Gamma \rightarrow \mathbf{H}^{3} / G$ is homotopic to a homeomorphism.

To prove Theorem 5.1, we need to make use of the following convergence theorem proved by Thurston in [34].

Theorem 5.2 (Thurston). Let $(N, X)$ be a pair of a compact 3-manifold whose interior admits a hyperbolic metric and a collection of simple closed curves on the boundary. Suppose that the following three conditions hold.

(1) There is no embedded essential annulus in $N$ whose boundary lies on $\partial N-X$.

(2) Any compression disc for $N$ intersects $X$ at no more than two points.

(3) Each maximal abelian subgroup of $\pi_{1}(\partial N-X)$ is mapped to a maximal abelian subgroup of $\pi_{1}(N)$ by the homomorphism induced from the inclusion. 
(We say that $(N, X)$ is doubly incompressible then.) Then for any constant $K$, the subset of $A H(N)$ consisting of the hyperbolic structures with respect to which the total geodesic length of $X$ is at most $K$ is compact. (The geodesic length of a closed curve representing $a$ parabolic element is defined to be 0 .)

Let $\gamma_{1}, \ldots, \gamma_{m}$ be a system of simple closed curves on $\Omega_{\Gamma} / \Gamma$ as is given in Theorem 5.1. We can assume that the simple closed curves are contained in $\partial \bar{M}_{0}$. Let $Y_{0}$ be the union of core curves of annulus components of $\operatorname{Fr} M_{0}=\partial \bar{M}_{0}-\Omega_{\Gamma} / \Gamma$, and let $Y$ be $\gamma_{1} \cup \ldots \cup \gamma_{m} \cup Y_{0}$. Since each core curve of an annulus component of $\operatorname{Fr} M_{0}$ represents a primitive parabolic class and none of $\gamma_{1}, \ldots, \gamma_{m}$ are freely homotopic to a closed curve representing a parabolic class, the assumption on $\gamma_{1}, \ldots, \gamma_{m}$ in Theorem 5.1 implies that the components of $Y$ satisfy the assumption of Proposition 4.1. Therefore we can extend it to a collection of simple closed curves satisfying the conclusion of Proposition 4.1, whose union we shall denote by $Z$. Now suppose that there is some component $\gamma$ of $Z$ which is not primitive. Since every component of $Y$ is primitive, such a component $\gamma$ is contained in $Z-Y$. In particular, such a $\gamma$ must represent a loxodromic class.

Then by Corollary 3.2, there exists a properly embedded essential annulus $A$ in $\bar{M}$ both of whose boundary components are parallel to $\gamma$ on $\partial \bar{M}=\Omega_{\Gamma} / \Gamma$. If two annuli corresponding to different components of $Z$ as above intersect, then the intersection can be assumed to consist of simple closed curves bounding discs on the annuli since none of the components of $Z$ are null-homotopic in $\bar{M}$ and no two distinct components of $Z$ are homotopic in $\bar{M}$. Thus by the cut-and-paste technique we can further assume that two annuli corresponding to distinct components of $Z$ are disjoint. Moreover we can isotope such an annulus so that it is contained in $\bar{M}_{0}$. Let $\Pi$ be the union of nonparallel disjoint essential annuli corresponding to non-primitive simple closed curves on the boundary as above. By Corollary 3.2, each component of $\Pi$ cuts off a solid torus from $M_{0}$. Let $N$ be a compact 3-manifold obtained by cutting $\bar{M}_{0}$ along $\Pi$ and removing solid torus components. Let $X$ be the union of $Z \cap N$ and the cores of components of $\Pi$.

Lemma 5.3. Let $P$ be the union of $\Pi$ and the torus components of $\operatorname{Fr} \bar{M}_{0}$. Then the pair $(N, P)$ is a pared manifold.

Proof. Let $T$ be either an embedded essential torus or a properly embedded essential annulus both of whose boundary components are in $\partial P$. It follows immediately from the definition of pared manifold that it suffices to prove that such a torus or an annulus is homotopic into $P$. We can see that $T$ and the closure of a component of $\operatorname{Fr} \bar{M}_{0}$ bound a submanifold $V$ homeomorphic to $T \times I$ in $\bar{M}_{0}$ because $\left(\bar{M}_{0}, \mathrm{Fr} \bar{M}_{0}\right)$ is a pared manifold. It is easy to see that $V \cap N$ gives rise to a homotopy from $T$ into $P$, which preserves the boundary when $T$ is an annulus. This is a contradiction.

Lemma 5.4. The pair $(N, X)$ above is doubly incompressible unless $N$ is a handle body of genus 2. 
Proof. We have to prove three conditions, that there is no embedded essential annulus whose boundary components are contained in $\partial N-X$, that any compression disc for $\partial N$ intersects $X$ at least in three points, and that every simple closed curve on $\partial N-X$ represents a generator of a maximal abelian group of $\pi_{1}(N)$. Since $N$ has no non-peripheral subgroup isomorphic to $\mathbf{Z} \times \mathbf{Z}$, the third condition is automatically satisfied if the first condition holds because then any simple closed curve in $\partial N-X$ is primitive by Lemma 3.1, and cannot by homotopic into a $\mathbf{Z} \times \mathbf{Z}$-cusp since it represents either a loxodromic element or a $Z$-cusp. Hence we have only to verify the first and second conditions.

First let $A$ be an embedded essential annulus in $\partial N-X$ whose boundary components we denote by $a$ and $b$. Since every component of $\partial \bar{M}_{0}-Z$ is homeomorphic to a thrice-punctured sphere, so is every component of $\partial N-X$. Hence $a$ and $b$ are homotopic to components $\alpha$ and $\beta$ of $X$ respectively in $\partial N$. No two components of $X$ are homotopic in $N$ because of the following reasons. First, no two components of $Z \cap N$ are homotopic in $N$ by the construction of $Z$. Secondly, no two cores of distinct components of $\Pi$ are homotopic by Lemma 5.3. Finally, no component of $Z \cap N$ can be homotopic to a core of a component of $\Pi$ since the former represents a loxodromic element while the latter represents a parabolic element. Thus we have $\alpha=\beta$. This means that there is an essential annulus in $N \subset \bar{M}_{0}$ whose boundary is parallel to $\alpha$, hence $\alpha$ is not primitive by Lemma 3.1. By the construction of $N$, all the components of $N \cap Z$ are primitive. It follows that $\alpha \subset X-Z$. By the definition of $N$ and $X$, there is a component $B$ of $\Pi$ whose boundary components are homotopic to $\alpha$ and which is an annulus on $\partial N$. We can assume that $\partial A=\partial B$ by isotoping $A$ in $N$. Let $C$ be an annulus on $\partial \bar{M}_{0}$ whose boundary is equal to $\partial A$. Since $N$ is atoroidal, $A \cup C$ bounds a solid torus $V$ which contains $B$. Then $B$ must be homotopic to either $A$ or $C$ in $V$ relatively to the boundary. Because $B$ is essential in $\bar{M}_{0}$, it cannot be homotopic to $C$, hence must be homotopic to $A$. It follows that $A$ is inessential in $N$, a contradiction.

Secondly suppose that there exists a compression disc $D$ which intersects $X$ at less than three points. Since every component of $\partial N-X$ is homeomorphic to a thricepunctured sphere and no component of $X$ is null-homotopic in $N$, the disc $D$ must intersect $X$. First consider the case when $D$ intersects $X$ at one point. Let $\gamma$ be the component of $X$ which intersects $D$. Let $P_{1}$ be a component of $\partial N-X$ whose closure contains $\gamma$. If there are two such components, then $\partial D$ must intersect $X$ at least twice. Hence $P_{1}$ is the unique component whose closure contains $\gamma$ and the closure of $P_{1}$ is a torus with a hole which contains $\partial D$. Now let $\beta$ be $\partial \bar{P}_{1}$, which is the only component of $\operatorname{Fr} P_{1}$ other than $\gamma$. Then after fixing a base point on $\partial D \cap \gamma$, the loop obtained from $\beta$ by connecting to the base point appropriately is homotopic to $\gamma[\partial D] \gamma^{-1}[\partial D]^{-1}$, hence must be contractible in $N$. This contradicts the fact the no components of $X$ are nullhomotopic in $N$.

Next consider the case when $D$ intersects $X$ at two points. By the argument in the last paragraph, we can assume that $\partial D$ is contained in the closure of the union of two components $P_{1}, P_{2}$ of $\partial N-X$. Let $P$ be the closure of $P_{1} \cup P_{2}$. There are five possibilities: the first is that $P$ is a sphere with four holes and $\partial D$ intersects twice a component $\gamma$ of $\boldsymbol{X}$ which separates $\boldsymbol{P}$ into two spheres with three holes; the second is 
that $P$ is a torus with two holes and $\partial D$ intersects two components $\gamma, \delta$ of $X$ both of which are contained in $P$ and whose union separates $P$ into two spheres with three holes; the third is that $P$ is a torus with two holes and $D$ intersects twice a component $\gamma$ of $X$, which is $\bar{P}_{1} \cap \bar{P}_{2}$, one of the closures of $P_{1}, P_{2}$ is a torus with a hole, and the other is a sphere with three holes; the fourth is that $P$ and the configuration of $\partial D$ and $\gamma$ are the same as in the third case, but both closures of $P_{1}, P_{2}$ are spheres with three holes, and some component of $\operatorname{Fr} P_{1}$ is also a component of $\operatorname{Fr} P_{2}$; and the fifth is that $P$ is a closed surface of genus 2. (See Figures.)

In the fifth case, by compressing $P$ along $D$, we get a torus $T$. This torus $T$ must be compressible since $N$ is atoroidal, which implies that $T$ bounds a solid torus. It follows that $\boldsymbol{P}$ bounds a handle body of genus 2 . By the same argument we can see that $\bar{M}$ is also a handle body of genus 2 because $\partial \bar{M}$ is a compressible closed surface of genus 2. This is the excluded case in our assumption. In the four cases from the first to the fourth, it follows that there must be two components of $\operatorname{Fr}\left(P_{1} \cup P_{2}\right)$ which are homotopic in $N$. This implies that there are two components $a, b$ of $X$ which are homotopic in $N$. Let $a^{\prime}, b^{\prime}$ be components of $Z$ as follows. If $a \subset Z$, then we let $a^{\prime}$ be $a$. If $a$ is not contained in $Z$, then there exists a component $a^{\prime}$ of $Z$ representing a non-primitive class lying on the boundary of a component of $\bar{M}_{0}-\Pi$ whose closure contains $a$. By definition, $a^{\prime}$ is freely homotopic to $a$. Similarly we define a component $b^{\prime}$ of $Z$ which is freely homotopic to $b$ unless $b$ is contained in $Z$. Since $a \neq b$, we have $a^{\prime} \neq b^{\prime}$. As $a$ and $b$ are freely homotopic in $N$, so are $a^{\prime}$ and $b^{\prime}$ in $\bar{M}_{0}$. This contradicts the construction of $Z$. (Recall that we constructed $Z$ so that no two components of $Z$ are homotopic in $\bar{M}_{0}$.) Thus we have proved that $(N, X)$ is doubly incompressible.

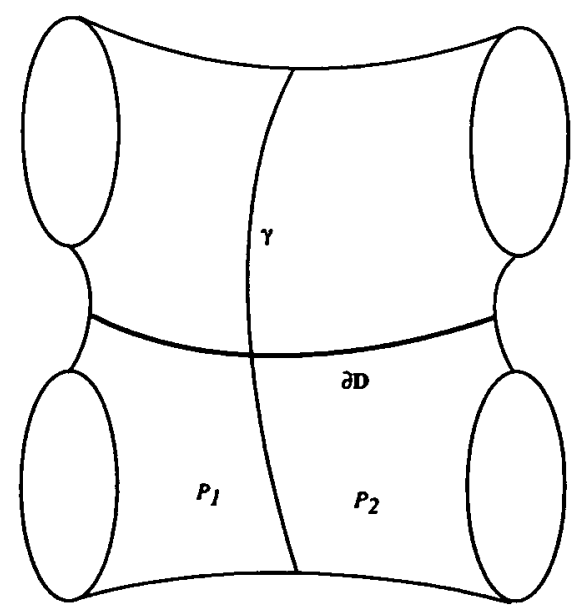

The first posability 


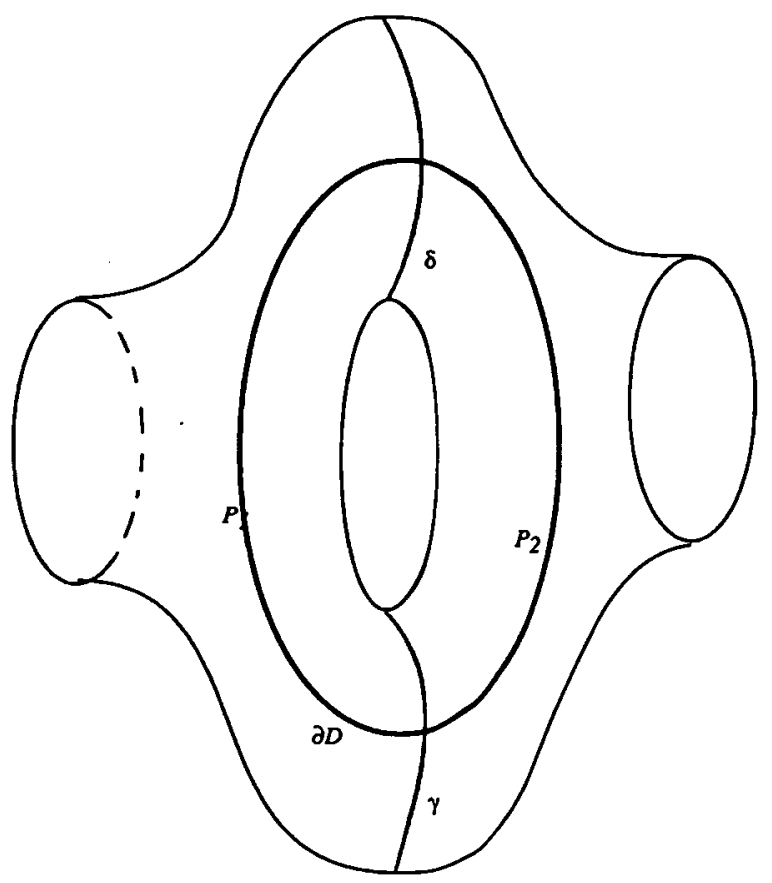

The second possibility

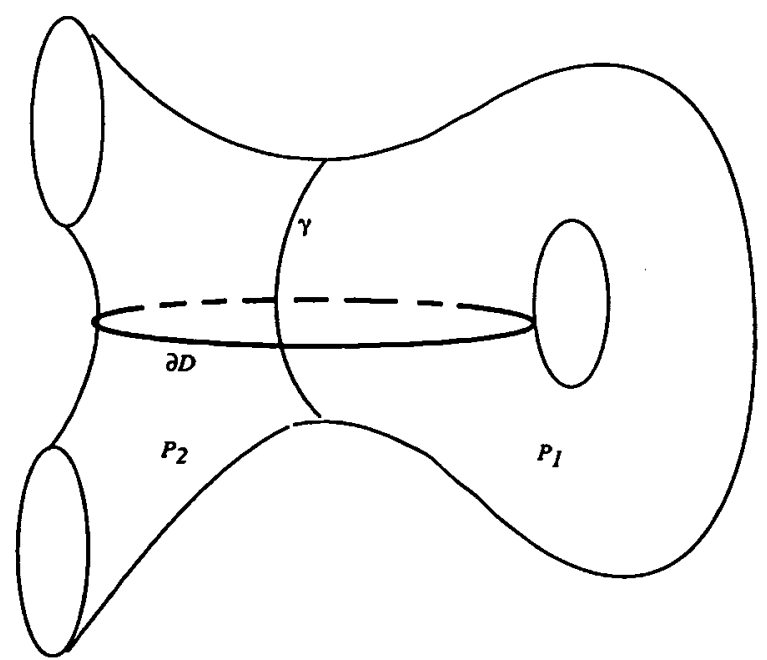

The third poseliblity 


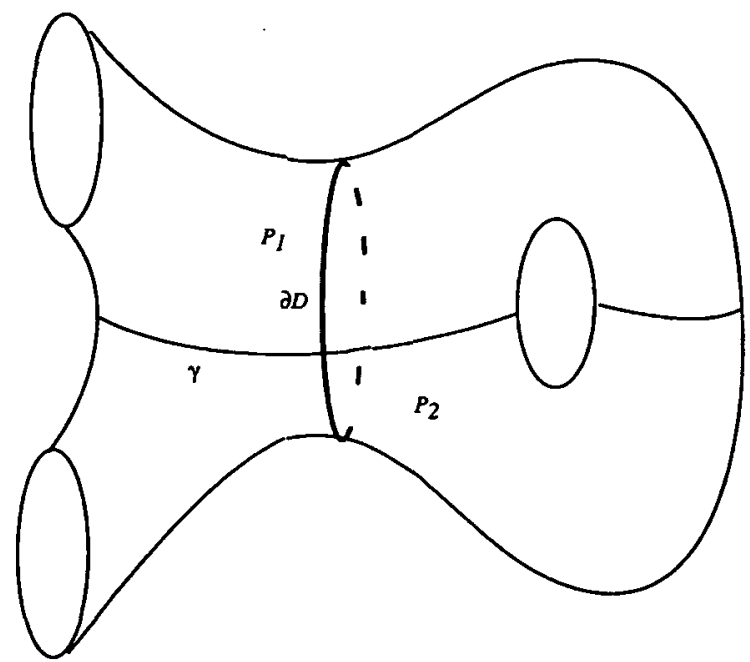

The fourth posesibility

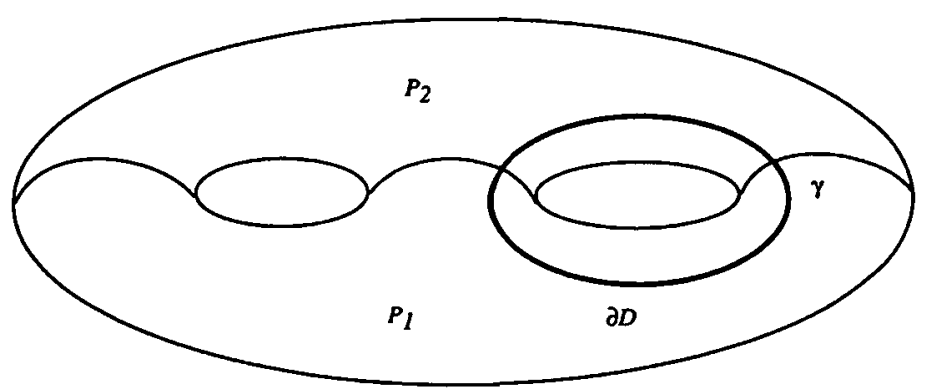

The fifh possibility

Proof of Theorem 5.1. Since Theorem 5.1 has already been proved in Maskit [15] when $\Gamma$ is a Schottky group, we can assume that $N$ is not a handle body of genus 2. (Note that $N$ is a handle body of genus 2 if and only if so is $\bar{M}_{0}$. This is equivalent to the condition that $\Gamma$ is a Schottky group of rank 2.) The conformal structure on $\Omega_{\mathrm{r}} / \Gamma$ induced from the conformal structure on the Riemann sphere $S_{\infty}^{2}$ determines a hyperbolic metric. Isotope $\gamma_{1}, \ldots, \gamma_{m}$ so that they are closed geodesics with respect to the hyperbolic metric. Let $g_{t}$ be a conformal structure on $\Omega_{\Gamma} / \Gamma$ 
obtained by a quasi-conformal deformation of the original conformal structure on $\Omega_{\Gamma} / \Gamma$, whose Beltrami coefficient is supported in the union of disjoint annulus neighbourhoods $A\left(\gamma_{1}\right), \ldots, A\left(\gamma_{m}\right)$ of $\gamma_{1}, \ldots, \gamma_{m}$ such that the extremal lengths of $\gamma_{1}, \ldots, \gamma_{m}$ with respect to $g_{i}$ are equal to $1 /(2 \pi i)$. We take the annulus neighbourhoods $A\left(\gamma_{1}\right), \ldots, A\left(\gamma_{m}\right)$ so that any component of $Z$ other than $\gamma_{1}, \ldots, \gamma_{m}$ is disjoint from $\cup A\left(\gamma_{j}\right)$, and we can assume that the geodesic length of $\bar{\gamma}_{k}$ for $k>m$ with respect to $g_{i}$ is equal to that with respect to the hyperbolic metric compatible with the original conformal structure on $\Omega_{\Gamma} / \Gamma$. Refer to Maskit [15] for further details to see how to construct such a quasi-conformal deformation.

As is shown in Bers [3], there is a map $q: \mathcal{T}\left(\Omega_{\Gamma} / \Gamma\right) \rightarrow Q H_{0}(\Gamma)$ such that for $g \in \mathcal{T}\left(\Omega_{\Gamma} / \Gamma\right)$, the quasi-conformal deformation $q(g)$ of $\Gamma$ is obtained by extending a Beltrami differential on $\Omega_{\Gamma}$ defined by $g$ to one on the entire $S^{2}$ by setting the value on $\Lambda_{\Gamma}$ to be 0. By Sullivan [29], it is known that any automorphic Beltrami differential supported on $\Lambda_{\Gamma}$ is trivial almost everywhere. This means that the map $q$ above is a surjection. Let $\left(\Gamma_{i}, \phi_{i}\right)$ be $q\left(g_{i}\right)$. Then we have length ${ }_{\mathrm{H}^{3} / \Gamma_{i}}\left(\phi_{i}\left(\left[\gamma_{j}\right]\right)\right) \leq 1 / i$ by Corollary 2 in Maskit [15]. On the other hand, for any component $\delta$ in $Z-\cup_{j=1}^{m} \gamma_{j}$, we have the equation length $g_{g_{t}}([\delta])=$ length $_{\Omega_{\Gamma} / \Gamma}(\delta)$ where the length ${ }_{\Omega_{\mathrm{T}} / \Gamma}$ means the hyperbolic length with respect to the hyperbolic metric compatible with the conformal structure on $\Omega_{\Gamma} / \Gamma$. By Corollary 3 in Maskit [16], the extremal length of $\delta$ with respect to $g_{i}$ is bounded as $i \rightarrow \infty$, which implies $\left\{\operatorname{length}_{\mathbf{H}^{3} / \Gamma_{i}}\left(\phi_{i}(\delta)\right)\right\}$ is bounded again by Corollary 2 in [15].

Now take a base-point in $N$, regard $\Gamma$ as $\pi_{1}(M)$, and let $\Gamma^{\prime}$ be a subgroup of $\Gamma$ which corresponds to $\pi_{1}(N)$ in $\pi_{1}(M)=\Gamma$. Let $\Gamma_{i}^{\prime}$ be $\phi_{i}\left(\Gamma^{\prime}\right)$ and $\phi_{i}^{\prime}$ the restriction of $\phi_{i}$ to $\Gamma^{\prime}$. The isomorphisms $\phi_{i}^{\prime}$ determine homotopy equivalences $\phi_{i}: N \rightarrow \mathbf{H}^{3} / \Gamma_{i}^{\prime}$. Hence $\left(\Gamma_{i}^{\prime}, \phi_{i}^{\prime}\right)$ can be regarded as an element of $A H(N)$. Then we can prove the following lemma.

Lemma 5.5. The sequence of Kleinian groups $\left\{\left(\Gamma_{i}^{\prime}, \phi_{i}^{\prime}\right)\right\}$ converges algebraically (in $A H(N))$ after taking a subsequence.

Proof. Let $\gamma$ be a component of $X$. Then $\gamma$ is either contained in $Z$ or freely homotopic to a component of $Z$ in $\bar{M}_{0}$. Therefore as is shown above, the geodesic length of $\phi_{i}([\gamma])$ in $\mathbf{H}^{3} / \Gamma_{i}$ is bounded above. Hence the length of $\phi_{i}^{\prime}([\gamma])$ in $\mathbf{H}^{3} / \Gamma_{i}^{\prime}$ is also bounded as $i \rightarrow \infty$. Thus Lemma 5.5. follows from Theorem 5.2 and Lemma 5.4.

Continuation of Proof of Theorem 5.1. Recall that $N$ is obtained from $\bar{M}_{0}$ by cutting off disjoint solid tori along $\Pi$. Let $V$ be one of the solid tori and let $N_{1}$ be the submanifold $N \cup V$ of $\bar{M}_{0}$. Let $\Gamma^{1}$ be a subgroup of $\Gamma$ associated to $\pi_{1}\left(N_{1}\right), \Gamma_{i}^{1}$ be $\phi_{i}\left(\Gamma^{1}\right)$, and $\phi_{i}^{1}$ be $\phi_{i} \mid \Gamma^{1}$. We shall prove that the fact that $\left\{\left(\Gamma_{i}^{\prime}, \phi_{i}^{\prime}\right)\right\}$ converges algebraically, proved in Lemma 5.5 , implies that so does $\left\{\left(\Gamma_{i}^{1}, \phi_{i}^{1}\right)\right\}$. We can take conjugates of the representations $\phi_{i}^{\prime}$ so that $\left\{\phi_{i}^{\prime}\right\}$ converges as a sequence of representations. Let $T$ be the boundary of $V$, which is a torus. Then $\pi_{1}(T)$ is generated by two elements $\alpha, \beta$ one of which, say $\alpha$, is represented by a core curve of an annulus in $\Pi$ along which $V$ is cut off from $\bar{M}_{0}$. Then the boundary of a meridonal disc of $V$ is homotopic to $\alpha^{p} \beta^{q}$, for integers $p, q$ with $q \neq 0$ because a core curve of an annulus in $\Pi$ 
cannot be the boundary of a meridonal disc. Since $\alpha^{p}$ is contained in $\pi_{1}(N)$, so is $\beta^{q}$. Therefore $\phi_{i}\left(\beta^{q}\right)$ converges as $i \rightarrow \infty$, which implies that so does $\phi_{i}(\beta)$. Hence $\left\{\left(\phi_{i} \mid \pi_{1}\left(N_{1}\right)\right)=\phi_{i}^{\prime}\right\}$ also converges as a sequence of representations.

By the same argument as above, we can prove that for a submanifold $N_{2}$ obtained by pasting to $N_{1}$ another solid torus which was cut off from $\bar{M}_{0}$, the sequence $\left\{\phi_{i} \mid \pi_{1}\left(N_{2}\right)\right\}$ converges. Repeating this in finite steps, we can prove that $\left\{\left(\Gamma_{i}, \phi_{i}\right)\right\}$ itself converges algebraically to a Kleinian group $(G, \psi) \in A H(\Gamma)$.

As length $\mathrm{H}^{3} / \mathrm{G}\left(\psi\left(\left[\gamma_{j}\right]\right)\right)=\lim _{i \rightarrow \infty}$ length $_{\mathrm{H}^{3} / \Gamma_{i}}\left(\phi_{i}\left(\left[\gamma_{j}\right]\right)\right)=0$, the conjugacy classes $\psi\left(\left[\gamma_{1}\right]\right), \ldots$, $\psi\left(\left[\gamma_{m}\right]\right)$ represent parabolic classes in $G$.

Let $\tilde{\gamma}_{j}$ be the inverse image of $\gamma_{j}$ in $\Omega_{\Gamma}$, and let $\tilde{A}\left(\gamma_{j}\right)$ be that of the annulus neighbourhood $A\left(\gamma_{j}\right)$. Since the quasi-conformal homeomorphism which we constructed to deform the conformal structure on $\Omega_{\Gamma} / \Gamma$ to $g_{i}$ is conformal outside $\cup A\left(\gamma_{j}\right)$, there is a quasi-conformal homeomorphism $\omega_{i}: S^{2} \rightarrow S^{2}$ which is conformal in $\Omega_{\Gamma}-\cup \tilde{A}\left(\gamma_{j}\right)$ such that $\omega_{i} \gamma \omega_{i}^{-1}=\phi_{i}(\gamma)$ for any $\gamma \in \Gamma$. Let $P$ be a component of $\Omega_{\Gamma} / \Gamma-\cup \gamma_{j}$. Let $\tilde{P}$ be a component of the inverse image of $P$ in $\Omega_{\Gamma}$, and let $\Gamma^{\tilde{p}}$ be the stabilizer of $\tilde{P}$ in $\Gamma$. Then there is a component $\tilde{P}^{\prime}$ of $\Omega_{\Gamma}-\cup A\left(\gamma_{j}\right)$ contained in $\tilde{P}$ which is also stabilized by $\Gamma^{\tilde{P}}$ since $U_{j} \tilde{A}\left(\gamma_{j}\right)$ is a $\Gamma$-invariant neighbourhood of $\cup_{j} \tilde{\gamma}_{j}$. Note that $\omega_{i}$ is conformal on $\tilde{P}$. By results in Section 4 in Maskit [15], there is an open set $Q$ of $\Omega_{G}$ which is stabilized by $\psi\left(\Gamma^{\bar{P}}\right)$. Then $Q / G$ is a subsurface of $\Omega_{G} / G$ whose punctures are freely homotopic to cusps of $\Omega_{G} / G$ freely homotopic to closed curves represented by $\psi\left(\gamma_{1}\right), \ldots, \psi\left(\gamma_{m}\right)$ in $\overline{\mathbf{H}}^{3} / G$. The homotopy equivalence $\psi: \mathbf{H}^{3} / \Gamma \rightarrow \mathbf{H}^{3} / G$ can obviously be extended to a homotopy equivalence $\bar{\psi}: \overline{\mathbf{H}^{3} / \Gamma} \rightarrow \overline{\mathbf{H}^{3} / G}$. Then what we have proved above implies that for each component $P$ of $\Omega_{\Gamma} / \Gamma-U \gamma_{j}$, there exists a component of $\Omega_{G} / G$ homotopic to $\bar{\psi}(P)$ in $\overline{\mathbf{H}^{3} / G}$ relatively to the punctures and the frontiers of $P$ if we first homotope $\bar{\psi}$ so that it takes the punctures and the frontiers of $P$ into cusps of $\mathbf{H}^{3} / G$.

We can assume that $\psi \mid \partial\left(\mathbf{H}^{3} / \Gamma\right)_{0}$ is an embedding into $\partial\left(\mathrm{H}^{3} / G\right)_{0}$ by homotoping $\psi$ because $\psi$ maps cusps to cusps. Let $S$ be a frontier component (in $\left(\mathbf{H}^{3} / \Gamma\right)_{0}$ ) of the convex core of $\left(\mathrm{H}^{3} / \Gamma\right)_{0}$. The surface $S$ corresponds to a component $\Sigma$ in $\Omega_{\mathrm{r}} / \Gamma$ and there is an embedding $\imath: S \rightarrow \Sigma$ which is a homotopy equivalence. (We can take the inverse of the "nearest point reaction". See Morgan [21].) Let $\hat{\gamma}_{1}, \ldots, \hat{\gamma}_{p}$ be disjoint simple closed curves on $S$ corresponding by $\imath$ to the simple closed curves among $\gamma_{1}, \ldots, \gamma_{m}$ which are contained in $\Sigma$. Then we can homotope $\psi$ first so that $\psi\left(\hat{\gamma}_{1}\right), \ldots, \psi\left(\hat{\gamma}_{p}\right)$ are core curves of $\partial\left(\mathrm{H}^{3} / G\right)_{0}$, and next so that $\psi\left(S-\cup_{j=1}^{p} \hat{\gamma}_{j}\right)$ is a homeomorphism onto a frontier component of the convex core of $\left(\mathbf{H}^{3} / G\right)_{0}$ preserving $\psi\left|\hat{\gamma}_{1}, \ldots, \psi\right| \hat{y}_{\mathrm{p}}$ by the fact proved in the last paragraph. This implies that the convex cores of $\left(\mathbf{H}^{3} / \Gamma\right)_{0}$ and of $\left(\mathbf{H}^{3} / G\right)_{0}$ are homeomorphic by a homeomorphism homotopic to $\psi$ by Waldhausen's theorem in [35], that $G$ is also geometrically finite, and that $\left[\psi\left(\gamma_{1}\right)\right]^{ \pm 1}, \ldots,\left[\psi\left(\gamma_{m}\right)\right]^{ \pm 1}$ are the only additional primitive parabolic classes. It also follows that $\psi$ is homotopic to a homeomorphism from $\mathbf{H}^{3} / \Gamma$ to $\mathbf{H}^{3} / G$. Finally, we can see that since $\left[\gamma_{j}\right]$ is primitive, so is $\psi\left(\left[\gamma_{j}\right]\right)$. 


\section{REFERENCES}

1. W. ABIxoff, On boundaries of Teichmüller spaces and on Kleinian groups III, Acta Math. 134 (1975), 211-237.

2. L. AHLFORS, Finitely generated Kleinian groups, Amer. J. Math. 86 (1964), 413-423.

3. L. BERS, Spaces of Kleinian groups (Lecture Notes on Mathematics 155, 1970), 9-34.

4. F. Bonamon, Bouts de variétés hyperboliques de dimension 3, Ann. of Math. 124 (1986), 71-158.

5. F. Bonahon, Cobordism of automorphisms of surfaces, Ann. Sci. Ecole Norm. Sup. (4) 16 (1983), 237-270.

6. R. D. Canary, D. B. A. Epstein and P. Green, Notes on notes of Thurston, Analytical and geometric aspects of hyperbolic spaces (London Math. Soc. Lecture Note Ser. 111, Cambridge Univ. Press, 1987), 3-92.

7. M. Culler and P. Shalen, Variety of group representations and splittings of 3-manifolds, Ann. of Math. 117 (1983), 109-146.

8. D. B. A. Epstern and A. MARden, Convex hulls in hyperbolic space, a theorem of Sullivan, and measured pleated surfaces, in Analytical and geometric aspects of hyperbolic spaces (London Math. Soc. Lecture Note Ser. 111, Cambridge Univ. Press, 1987), 113-253.

9. J. Hempel, 3-Manifolds (Ann. of Math. Studies, No. 86. Princeton Univ. Press).

10. W. JACo, Lectures on three-manifold topology (CBMS regional conf. ser. in math. 43, 1980).

11. W. JACo and P. Shalen, Seifert fibered spaces in 3-manifolds (Mem. Amer. Math. Soc. 21, 1979).

12. T. Jørgensen, On discrete groups of Möbius transformations, Amer. J. Math. 98 (1976), 739-749.

13. T. Jørgensen and A. MARden, Algebraic and geometric convergence of Kleinian groups, Math. Scand. 66 (1990), 47-72.

14. A. Marden, The geometry of finitely generated Kleinian groups, Ann. of Math. 99 (1974), 465-496.

15. B. MASKIT, Parabolic elements on Kleinian groups, Ann. of Math. 117 (1983), 659-668.

16. B. Maskir, Comparison of hyperbolic length and extremal length, Ann. Acad. Sci. Fenn. Ser. A I Math. 10 (1985), 381-386.

17. B. MASKIT, Kleinian groups (Grundlehren der mathematischen Wissenschaften 287, Springer-Verlag, Berlin-Heidleberg, 1988).

18. D. McCullough, Compact submanifolds with boundary, Quart. J. Math. Oxford Ser. (2) 37 (1986), 299-307.

19. W. Meeks III and S. T. YAU, The classical Plateau problem and the topology of threedimensional manifolds. The embedding of the solution given by Douglas-Morrey and an analytic proof of Dehn's lemma, Topology 21 (1982), 409-442.

20. D. McCullough, A. Miller and G. A. Swarup, Uniqueness of cores of non-compact 3-manifolds, J. London Math. Soc. 32 (1985), 548-556.

21. J. MORGan, On Thurston's uniformization theorem for three-dimensional manifolds, in The Smith Conjecture (Academic Press, New York, London, 1984), 37-125. 
22. J. MORGaN and P. SHALEN, Degeneration of hyperbolic structures III, action of 3-manifold groups on trees and Thurston's compactness theorem, Ann. Math. 120 (1988), 457-519.

23. K. OHSHIKA, Ending laminations and boundaries for deformation spaces of Kleinian groups, J. London Math. Soc. (2) 42 (1990), 111-121.

24. K. OнSнIKA, Geometric behaviour of Kleinian groups on boundaries for deformation spaces, Quart. J. Math. Oxford (2) 43 (1992), 97-111.

25. K. Онsнira, Parabolization of elements of Kleinian groups, in Topology and Teichmüller space (World Scientific, 1996), 221-236.

26. G. P. Scotr, Compact submanifolds of 3-manifolds, J. London Math. Soc. (2) 7 (1973), 246-250.

27. A. Shapiro and J. H. C. Whitehead, A proof and extension of Dehn's lemma, Bull. Amer. Math. Soc. 64 (1958), 174-178.

28. J. Stallings, On the loop theorem, Ann. of Math. 72 (1960), 12-19.

29. D. Sullivan, On the ergodic theory at infinity of an arbitrary discrete group of hyperbolic motions, in Riemann Surfaces and Related Topics (Ann. Math. Studies 97, Princeton Univ. Press, 1980), 465-498.

30. D. Sullivan, A finiteness theorem for cusps, Acta Math. 147 (1980), 289-299.

31. W. Thurston, The geometry and topology of 3-manifolds (Lecture notes, Princeton Univ.).

32. W. Thurston, Hyperbolic structures on 3-manifolds I: Deformation of acylindrical manifolds, Ann. of Math. 124 (1986), 203-246.

33. W. THurston, Hyperbolic structures on 3-manifolds II: Surface groups and 3-manifolds which fiber over the circle, preprint, Princeton Univ.

34. W. Thurston, Hyperbolic structures on 3-manifolds III: Deformations of 3-manifolds with incompressible boundary, preprint, Princeton Univ.

35. F. Waldhausen, On irreducible 3-manifolds which are sufficiently large, Ann. of Math. 87 (1968), 56-88.

Department of Mathematical Sciences

UNIVERSITY OF TOKYO

KOMABA

TORYO 153

JAPAN 